\title{
BIM WD: A DIMENSÃO DOS TRABALHADORES INTEGRADA NOS MODELOS DE INFORMAÇÃO
}

\author{
Diego Calvetti (1), Luís Sanhudo ${ }^{(1)}$, Pedro Mêda ${ }^{(2)}$, Miguel Gonçalves ${ }^{(1)}$, João Poças \\ Martins $^{(1)}$, Hipólito Sousa ${ }^{(1)}$, Nuno M. M. Ramos ${ }^{(3)}$ \\ (1) CONSTRUCT-Gequaltec, Faculdade de Engenharia da Universidade do Porto, Porto \\ (2) CONSTRUCT-Gequaltec, Instituto da Construção, Faculdade de Engenharia da \\ Universidade do Porto, Porto \\ (3) CONSTRUCT-LFC, Faculdade de Engenharia da Universidade do Porto, Porto
}

\begin{abstract}
Resumo
Adicionar "dimensões" ou informação a modelos BIM tem o potencial de ampliar a compreensão sobre um projeto de construção. As dimensões BIM retratam a forma como esta metodologia é aplicada a todo o ciclo de vida da construção, nomeadamente: 4D (temporal); 5D (financeira); 6D (sustentabilidade); 7D (gestão das instalações); para além das três dimensões do espaço euclidiano.

A segurança em obra e a produtividade na indústria da construção podem ser melhoradas a partir da análise detalhada das tarefas realizadas no estaleiro. Os avanços tecnológicos, designadamente os dispositivos vestíveis (Wearables), propiciam novas formas de recolher este tipo de informação, enquanto que o recurso à inteligência artificial permite agilizar a sua análise. Estudos em desenvolvimento no CONSTRUCT/Gequaltec (FEUP) recorrem a dispositivos electrónicos para a colheita de dados relativos aos movimentos dos trabalhadores da construção. À posteriori, a análise de dados é efectuada por meio de algoritmos baseados em aprendizagem computacional. Os resultados desta análise permitem monitorizar a ocupação dos trabalhadores durante as suas atividades diárias (e.g. serrar, pintar, caminhar, descansar), permitindo uma avaliação detalhada dos processos produtivos, e demonstrando um elevado potencial para gestão de obras, em particular nos domínios da segurança e do controlo da produtividade. Conceitualmente, estes novos dados são processados por meio de tecnologias e técnicas de informação, sendo posteriormente integrados no BIM e dando origem a uma nova dimensão, aqui denominada WD Workers Dimension.

Apresenta-se neste artigo os conceitos e modelos desta nova dimensão/uso BIM, potenciados pela utilização de Wearables e a aplicação de Machine Learning.
\end{abstract}

\section{Introdução}

A Indústria da Construção (IC) mobiliza mundialmente em média USD 10e12 por ano, tornando-a num dos maiores influenciadores da economia mundial [1]. Contudo, o crescimento 
da produtividade dentro da IC é inferior à média das restantes indústrias. De facto, nas últimas duas décadas o crescimento da produtividade industrial foi aproximadamente $3,6 \%$ ao ano, face a um crescimento mais modesto de $1 \%$ por parte da IC [1]. Adicionalmente, nos próximos anos, a força de trabalho disponível no Reino Unido poderá sofrer um declínio de 20 a 25\% [2]. Contudo, os efeitos desta redução poderão ser mitigados mediante a aplicação de inovações para aumentar a produtividade do trabalho nos estaleiros [2].

As empresas em tecnologia da construção investiram, em termos mundiais, USD 10e9 por ano em software, hardware e recursos de análise nos últimos 10 anos [3]. Soluções tecnológicas inovadoras para a indústria da construção precisam oferecer uma experiência contínua e em tempo real, como exemplo no campo da [4]: Gestão de projetos, com a integração 3D, 4D e 5D por meio de plataformas móveis [4]; Gestão de contratos, com comunicação de informações da obra em tempo real [4]; Gestão de materiais, identificação e rastreamento de materiais e equipamentos [4]; Controlo da qualidade, com inspeção remota com o uso de imagens e etiquetas (radiofrequência) [4]; Monitorização da produtividade dos trabalhadores em tempo real [4].

Sendo a construção uma indústria fortemente influenciada pelos seus recursos humanos, a monitorização dos trabalhadores é vital. Vários fatores positivos e negativos influenciam direta e indiretamente a produtividade do trabalhador. Nomeadamente, um trabalhador saudável num ambiente seguro está propenso a atingir um melhor desempenho, além de ter um menor risco de sofrer ações externas que resultem em improdutividade. A fadiga dos trabalhadores da construção está diretamente relacionada à falta de produtividade, perda na qualidade dos serviços e aumento do risco de acidentes [5].

Pretende-se com este trabalho indicar uma forma de automatizar a recolha e tratamento da informação relativa à produtividade dos trabalhadores e estruturá-la aos vários usos BIM associando-se estes dados à dimensão denominada de WD Workers Dimension.

\section{Referencial Teórico}

\subsection{BIM (Dimensões/Usos)}

O conceito de "multi-dimensões" BIM (multi-dimensional BIM - nD BIM) [6-7] consiste no uso de informação contida em modelos desta metodologia, para uma melhor gestão de projeto ao longo do seu ciclo de vida (e.g. tempo e custo). Atualmente, na literatura, pode-se identificar uma falta de consenso no que diz respeito à nomenclatura associada aos diversos usos da informação BIM, particularmente no significado das novas dimensões. Neste sentido, um questionário realizado em 28 países europeus teve como objetivo fazer uma consolidação do significado de cada dimensão e respetivo tópico associado. As respostas demonstraram uma discrepância no que diz respeito às dimensões 6D e 7D, sendo que estas foram simultaneamente associadas aos tópicos de Sustentabilidade, Gestão de Instalações, e Segurança [8]. De facto, os resultados indicam que profissionais praticantes destas dimensões referem-se às atividades de Sustentabilidade como 6D (86\%) e Gestão de Instalações como 7D (85\%) [8], enquanto que profissionais não praticantes destas dimensões fazem o relacionamento inverso. Não obstante, acentua-se neste trabalho e na atual industria, a falta de integração de informação relativa aos trabalhadores de mão de obra (WD) nas dimensões BIM, existindo o potencial de fazer esta integração por forma a melhorar a gestão de projetos. 


\subsection{Monitorização autónoma do desempenho}

$\mathrm{O}$ atual avanço tecnológico proporciona uma gama de diferentes dispositivos para colheita de dados em estaleiro, nomeadamente: aparelhos fotográficos e de filmagem, dispositivos de radiofrequência, telemóveis e relógios inteligentes. Estes dispositivos electrónicos devem ser selecionados com base numa análise de custo-benefício, tendo como base principal o que se pretende monitorizar. De realçar que a combinação de diferentes tecnologias pode trazer resultados mais efetivos e mais econômicos. Devido à complexidade de implementação a monitorização electrónica exige recursos especializados. A automação do processo de aquisição de dados relativos a análise da segurança e produtividade do trabalhador, focando-se na monitorização de fatores como: localização; trajetória; movimentos e gestos; tempo de ação; fisiologia; fatores externos (e.g. temperatura, pressão, humidade); entre outros. [9]

Neste trabalho, os resultados apresentados são referentes à colheita de dados por dispositivos wearable, sobre a forma de acelerómetros, usados em ambos os pulsos e no tornozelo da perna dominante dos indivíduos em análise. Destaca-se que, por estarem próximas do corpo humano, estas tecnologias devem possuir anatomia e segurança adequadas, como por exemplo, evitar choques elétricos ou queimaduras [10]. Outras questões importantes são: estética, tamanho, resistência a intempéries e ambientes agressivos, consumo de energia, capacidade de comunicação wifi e usabilidade em relação ao sistema operacional utilizado [10].

Tendo em vista a grande quantidade de dados coletados por meio dos dispositivos electrónicos e com o intuito de processar esta informação de forma rápida e autónoma, torna-se necessária a aplicação de processos baseados em aprendizagem computacional. Estudos académicos com foco no reconhecimento de atividades/ações humanas passaram pelo desenvolvimento de algoritmos baseados em Machine Learning [11-13] e seu subtipo o Deep Learning [14-15]. Como classificadores baseados em Machine Learning, pode-se destacar a aplicação de Decision Tree (DT), Discriminant Analysis (DA), Support Vector Machine (SVM), $k$-Nearest Neighbour (kNN), Nä̈ve Bayes (NB), Multilayer perceptron (MP) [14], [16-17]. E o método de Convolutional Neural Network (CNN) [14] em Deep Learning.

\section{Método aplicado}

No desenvolvimento dos modelos e conceitos, Modelo de colheita; Modelo de processamento e; Modelos de informação e gestão, apresentados no parágrafo 4. neste trabalho foi utilizada a técnica Delphi em conjunto com o método de grupo de discussão. Pretende-se, com estas duas metodologias de trabalho abordar a forma de estruturar a informação relativa à dimensão WDtrabalhadores nos usos BIM. Ainda, são apresentados resultados de experimentos realizados em laboratório na simulação de atividades da construção civil com o posterior tratamento de dados por meio de Machine Learning. A técnica Delphi é utilizada para obter o consenso independente entre especialistas por meio de rodadas de questões [18-19]. Grupos de discussão (Focus Group) reúnem partes interessadas e especialistas para uma discussão interativa sendo usualmente utilizadas para coleta de dados e tomadas de decisão [20].

Para o processo de desenvolvimento e validação dos modelos e conceitos apresentados neste artigo foi realizado um processo iterativo entre os autores e especialistas consultados conforme os passos descritos a seguir: (a) Delphi, Questionários (via Google Form) contendo um esboço dos modelos e conceitos; (b) Grupo de discussão, primeira avaliação. Reunião entre dois dos autores para análise e consolidação das primeiras respostas ao questionário e, desenvolvimento 
do segundo questionário; (c) Por fim, todo o artigo, contendo os modelos e a parte textual, foi compartilhado em modo de revisão entre os autores para discussão e consolidação final.

\section{Conceitos, Modelos e Trabalhos Desenvolvidos}

\subsection{O Processo WD Workers Dimension}

O processo autónomo aqui conceituado para análise do desempenho dos trabalhadores da mão de obra direta da indústria da construção é baseado em quatro subprocessos, conforme apresentado na Figura 1. Primeiramente deve ser realizada a colheita de dados em campo através da sensorização dos trabalhadores durante o desenvolvimento das suas atividades laborais. Concomitantemente, os modelos de processamento de dados são aplicados para obtenção dos resultados. posteriormente as informações tratadas serão armazenadas nos bancos de informação associadas aos usos BIM. Por fim, com base nestas informações os modelos de gestão são operacionalizados. Na sequência apresenta-se estes subprocessos em três itens: Modelo de colheita; Modelo de processamento e; Modelos de informação e gestão.

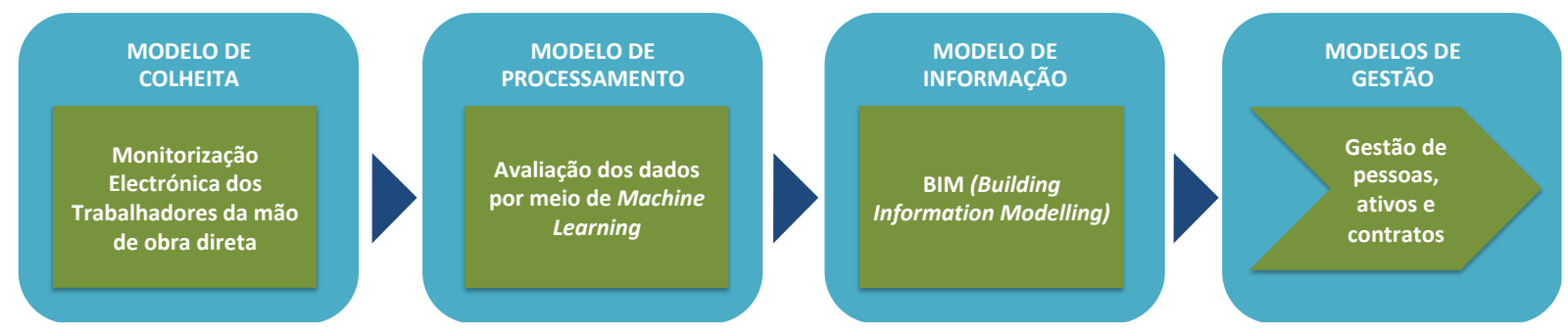

Figura 1: Processo de gestão na dimensão do Trabalhador.

\subsection{Colheita de Dados para Avaliação do Desempenho na Construção}

A metodologia para colheita de dados por meio de dispositivos vestíveis baseia-se em hardware e software, vide Figura 2. Os elementos "físicos" (hardware) são associados aos trabalhadores propriamente ditos quando estes vestem os dispositivos durante as suas rotinas laborais. De referir ainda toda a infraestrutura física indispensável de dispositivos e equipamentos para transmitir, armazenar e processar os dados. Associados aos dispositivos e equipamentos encontram-se os sistemas (softwares) nativos para operação/funcionamento dos mesmos. Por fim, são necessários diferentes sistemas (softwares) para armazenar e processar os dados como exemplo os sistemas operacionais e os bancos de dados.

Dentro deste conceito, e à luz da dimensão do trabalhador, pressupõe-se que as análises e os resultados terão o principal impacto nos campos da Segurança, Produtividade e Qualidade, Figura 3. A monitorização permite melhorar o comportamento dentro do estaleiro em relação aos projetos de prevenção de acidentes e mapeamento de riscos. Conhecer a produtividade e a fadiga dos funcionários dentro das atividades permite melhorar a produção e a segurança. A qualidade estará relacionada com a melhoria da produtividade quanto aos tempos e jornadas de trabalho, bem como detetar atempadamente a fadiga para que o serviço possa ser interrompido antes que seja afetado pela desatenção.

Existe uma clara relação entre a dimensão dos trabalhadores, a produtividade e a segurança dos mesmos, bem como a qualidade do trabalho por eles desempenhado. Esta influência é muito significativa, sendo que existem vários aspectos que podem beneficiar dela, nomeadamente: 
alertas de perigo e acidente para o trabalhador e equipa de segurança; obtenção de indicadores para o auxílio na melhoria da eficiência e produtividade em obra; entre outros.

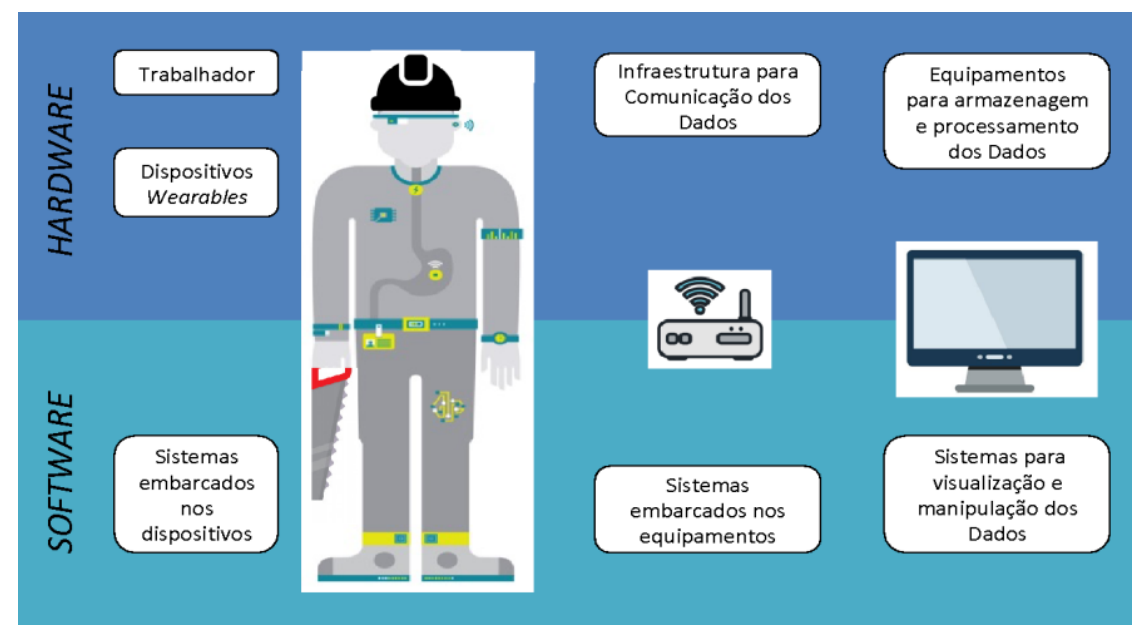

Figura 2: Metodologia para colheita de dados baseada em hardware e software.

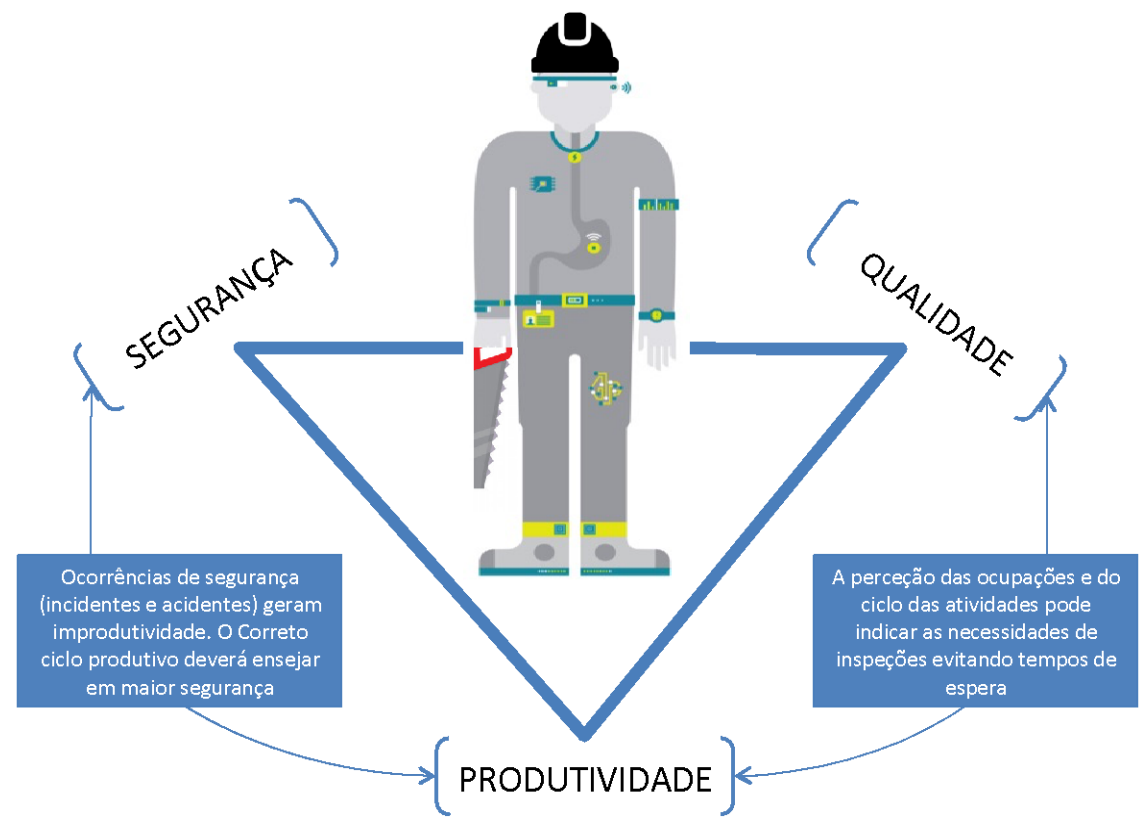

Figura 3: Dimensão do trabalhador.

Foram realizados ensaios no laboratório CONSTRUCT/Gequaltec (FEUP) com o objetivo de testar a viabilidade do uso de acelerómetros na colheita de dados para reconhecimento da atividade dos trabalhadores da construção, conforme fotos apresentadas na Figura 4. Os dados foram coletados ao longo de um percurso com dez atividades, com a utilização de três dispositivos dotados de acelerómetros. Um total de seis voluntários vestiram os dispositivos em ambos os pulsos e no tornozelo da perna dominante ao realizarem um circuito de atividades, a saber: (1) vestir EPIs; (2) martelar; (3) elevar alvenaria; (4) pintar; (5) simular o ato de chapiscar; (6) serrar com ferramenta manual; (7) aparafusar com ferramenta manual; (8) ficar sentado; (9) ficar parado; e (10) andar. 

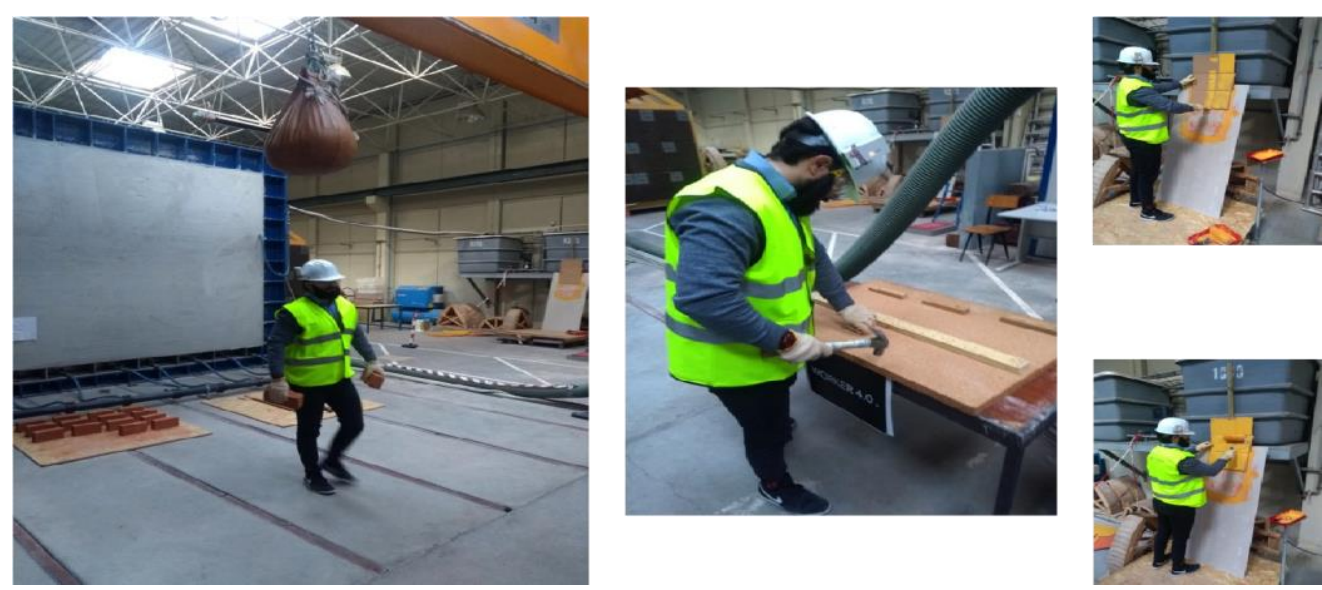

Figura 4: Imagens de alguns experimentos realizados.

\subsection{Processamento de Dados por meio de Machine Learning}

Como foi anteriormente referido, a automatização do processamento de dados é vital para a efetividade de um sistema capaz de manipular um grande volume de informação dentro de um pequeno tempo de resposta. O processo indicado está representado na Figura 5. Após a colheita dos dados, todos os pontos de informação devem ser caracterizados manualmente, pelo que se indica a necessidade de fazer uso de gravações de vídeo como referência. Estes pontos são a identificação/rótulo do tipo de processo/acção (ex., pregar, ajustar, caminhar, parado) no exato momento temporal da análise. De seguida realiza-se o processo de segmentação dos dado, sendo estes comummente divididos em grupos conforme as características da amostra. De seguida faz-se a extração das "features" que são as características específicas das amostras. Posteriormente são eleitas tais características que serão utilizadas como parâmetros para a classificação dos grupos de amostras. Por fim, são selecionados os classificadores (ex. Decision Tree, Discriminant Analysis, Support Vector Machine) e realizada a medição do desempenho/fiabilidade $(\%)$ de cada método relativamente à observação/identificação da amostra. Por fim, estabelecido este processo, pode-se indicar um conjunto de algoritmos para a realização de futuras análises autónomas para amostras semelhantes.

Para o processamento dos dados coletados no experimento apresentado na secção anterior, as dez atividades foram divididas em três grupos, sendo: Grupo 1 (G1) Elevar Alvenaria, Pintar, simular o ato de chapiscar; Grupo 2 (G2) Martelar, Serrar, Parafusar; Grupo 3 (G3) Vestir EPIs, Ficar Sentado, Ficar Parado, Andar. Várias condições de classificação foram estudadas, incluindo: o tamanho ideal da janela temporal (em segundos) para segmentar os dados; a extração e seleção de características artificiais relevantes; o ajuste dos hiperparâmetros (janelas de tempo e pastas de agrupamento dos parâmetros); e no final o treino e a seleção do classificador com melhor desempenho através de uma abordagem de validação cruzada.

Finalmente, os classificadores foram aplicados numa avaliação independente (sem treino do algoritmo) e dependente (com treino prévio do algoritmo) do sujeito para todas as atividades, com a utilização da janela de tempo ótima selecionada. Treze classificadores diferentes foram aplicados. Isso inclui oito modelos básicos e cinco métodos de conjunto, a saber: Modelos básicos (Base models): Decision Tree (DT); K-Nearest Neighbours (KNN); Logistic Regression (LR); Multilayer Perceptron (MLP); Multiclass Support Vector Machines (SVM) com diferentes "kernels" (linear (LSVM), polynomial (PSVM), radial basis function - rbf (RSVM), sigmoid (SSVM)). Métodos de conjunto (Ensemble methods): Random Forest (RF); Extremely 
Randomized Trees (ExT); AdaBoost (AdB); Gradient Boosting (GrB); Majority/Hard Voting (Vote).
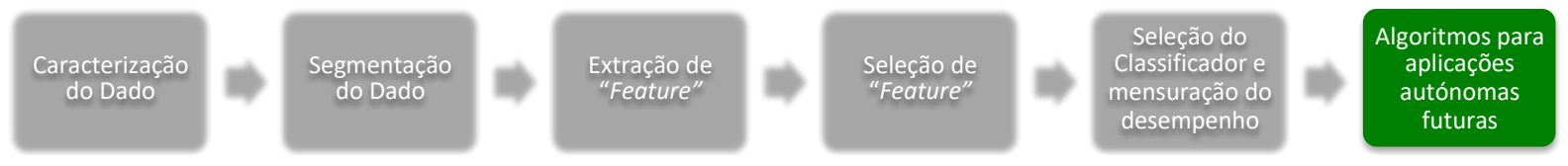

Figura 5: Automação do processamento de dados.

Para a abordagem de avaliação independente do sujeito (subject-independent) foi aplicada para cada grupo de atividades as janelas com diferentes tempos (4, 5 ou 6 segundos), vide Tabela 1 . Em relação ao desempenho das janelas, em 4 segundos (J4s) obteve-se um desempenho consideravelmente pior quando comparado à $\mathrm{J} 5 \mathrm{~s}$ e $\mathrm{J} 6 \mathrm{~s}$, com um desempenho médio em $\mathrm{J} 4 \mathrm{~s}$ (G1 85,20\%; G2 93,10\%; G3 90,63\%) contra J5s (G1 86,17\%; G2 93,10\%; 91,24\%) e por fim J6s (G1 87,54\%; G2 93,52\%; 91,49\%). Quanto aos classificadores, o melhor desempenho médio geral foi alcançado pelo Vote $92,34 \%$, seguido respetivamente por $A d B$ (91,58\%), GrB $(91,44 \%)$.

A avaliação independente contida na Tabela 2 evidencia o desempenho dos classificadores ao prever todas as dez atividades com a janela ideal (6 segundos). Uma abordagem dependente do sujeito (subject-dependent) foi usada para verificar quanto do desempenho do classificador beneficiaria com o treino de novos sujeitos (trabalhadores) antes de prever as suas atividades. Essa abordagem foi aplicada apenas a todas as atividades com a janela ideal, vide Tabela 2. Como foi observado, o desempenho médio geral para a abordagem dependente do sujeito aumentou cerca de $6 \%$ em relação à abordagem independente. Por consequência destaca-se como potencial para uma sistematização autónoma os resultados de diagnóstico das dez atividades dos trabalhadores com fiabilidade média-máxima respetivas de: 86\%-94\% mediante uma calibração prévia (trabalhador executaria um circuito teste) ou; $80 \%-86 \%$ sem a realização de calibrações.

Tabela 1: Análise por grupos e janelas

\begin{tabular}{|c|c|c|c|c|c|c|c|c|c|c|c|c|c|c|c|}
\hline \multirow{2}{*}{ Grupo } & \multirow{2}{*}{ Janela } & \multicolumn{13}{|c|}{ Classificadores, desempenho médio (\%) } & \multirow{2}{*}{$\begin{array}{c}\text { Média } \\
\text { (\%) }\end{array}$} \\
\hline & & $D T$ & KNN & $L R$ & MLP & LSVM & PSVM & RSVM & SSVM & $R F$ & ExT & $A d B$ & GrB & Vote & \\
\hline \multirow{3}{*}{ G1 } & J4s & 81,25 & 80,44 & 86,44 & 86,63 & 87,41 & 86,14 & 87,05 & 86,06 & 83,26 & 83,75 & 85,54 & 84,98 & $88,80^{*}$ & 85,20 \\
\hline & J5s & 78,96 & 82,99 & 88,18 & $89,96^{*}$ & 88,72 & 85,70 & 87,10 & 85,27 & 87,10 & 84,19 & 87,87 & 85,84 & 88,27 & 86,17 \\
\hline & J6s & 84,00 & 82,42 & 90,03 & 82,85 & $92,71^{*}$ & 87,14 & 88,31 & 88,23 & 86,67 & 86,22 & 89,06 & 90,85 & 89,52 & 87,54 \\
\hline \multirow{3}{*}{ G2 } & $J 4 s$ & 89,83 & 91,77 & 94,91 & 94,04 & $95,74^{*}$ & 93,07 & 94,51 & 94,51 & 89,42 & 91,98 & 92,06 & 93,14 & 95,32 & 93,10 \\
\hline & J5s & 89,09 & 91,69 & 95,94 & 95,20 & 95,80 & 93,26 & 95,20 & 94,32 & 95,50 & 92,56 & 93,81 & 93,71 & $96,07^{*}$ & 94,01 \\
\hline & J6s & 91,99 & 93,22 & 93,46 & 95,15 & 92,64 & 93,75 & 94,11 & 94,02 & 93,11 & 93,25 & 93,16 & 93,18 & $94,78^{*}$ & 93,52 \\
\hline \multirow{3}{*}{ G3 } & $J 4 s$ & 93,31 & 93,49 & 85,68 & 86,27 & 84,55 & 89,14 & 91,23 & 85,28 & 93,86 & 94,13 & $94,58^{*}$ & 93,59 & 93,04 & 90,63 \\
\hline & $\mathrm{J} 5 \mathrm{~s}$ & 93,38 & 93,57 & 86,36 & 91,38 & 87,05 & 92,65 & 88,69 & 86,76 & 93,33 & $93,88^{*}$ & 93,80 & 93,05 & 92,22 & 91,24 \\
\hline & J6s & 92,89 & 92,82 & 84,14 & 85,34 & 86,84 & 92,73 & 94,52 & 90,25 & 94,04 & 93,77 & 94,38 & $94,66^{*}$ & 93,04 & 91,49 \\
\hline \multicolumn{2}{|c|}{ Média } & 88,30 & 89,16 & 89,46 & 89,65 & 90,16 & 90,40 & 91,19 & 89,41 & 90,70 & 90,41 & 91,58 & 91,44 & $92,34 *$ & 90,32 \\
\hline
\end{tabular}

* valores máximos 
Tabela 2: Análise independente e dependente do sujeito

\begin{tabular}{|c|c|c|c|c|c|c|c|c|c|c|c|c|c|c|}
\hline \multirow{2}{*}{ Avaliação } & \multicolumn{13}{|c|}{ Classificadores, desempenho médio (\%) } & \multirow{2}{*}{$\begin{array}{c}\text { Média } \\
\text { (\%) }\end{array}$} \\
\hline & $D T$ & $K N N$ & $L R$ & MLP & LSVM & PSVM & RSVM & SSVM & $R F$ & ExT & $A d B$ & GrB & Vote & \\
\hline Independente & 72,91 & 80,46 & 76,11 & 74,16 & 77,38 & 82,16 & 83,03 & 79,65 & 83,62 & 82,78 & 83,40 & $85,54^{*}$ & 83,84 & 80,39 \\
\hline Dependente & 78,14 & $93,69 *$ & 78,10 & 80,78 & 75,82 & 87,58 & 86,57 & 78,91 & 90,00 & 92,24 & 93,57 & 92,54 & 91,08 & 86,08 \\
\hline
\end{tabular}

\subsection{Integração com os Modelos de Informação e Gestão}

Por fim, as informações referentes à dimensão do trabalhador (WD - Worker Dimension) coletadas e processadas devem ser integradas no BIM e utilizadas na gestão dos projetos. É fundamental definir uma estrutura e forma de armazenamento e gestão dos dados relativos aos trabalhadores para apoiar e gerenciar os dados não gráficos nos modelos BIM.

Relativamente à visualização (3D), a informação a integrar passa pela avaliação das áreas de risco e zonas potenciais de ocorrência de incidentes e acidentes. Poderá ainda agregar-se a informação e visualização das zonas de ocupação ou de não produtividade dos trabalhadores diretos. Uma informação importante a agregar-se será a relativa ao estado das inspeções e rastreabilidade dos elementos construídos associados à ocupação dos inspetores e à avaliação consequente do nível de qualidade atingida.

A incorporação de informação no tempo (4D) e custo (5D) é possível ser feita de forma integrada por estarem intrinsecamente ligadas. Indubitavelmente, o aumento da produtividade está diretamente relacionado com melhores desempenhos em tempo e custo. Analisar o dispêndio dos recursos ao longo do processo de execução pode influenciar na decisão de alteração do processo construtivo ou até indicar novas alternativas no projeto (design). Por exemplo, na construção de um edifício com andares tipo, pode-se ao observar um elemento com baixo desempenho em tempo e custo, efetuar alterações nos próximos andares. Esta ideia está associada a dois conceitos cada vez mais destacados na indústria da construção, notadamente a "Construtibilidade" (Constructability) e a "Replicação" (Digital twins).

No que se refere à sustentabilidade (6D) aumentos em eficiência produtiva podem levar a um menor desperdício económico e de material em obra. Quanto à gestão das instalações (7D), tendo em conta que é uma tendência no mercado da construção o incremento do número de pequenas empresas e profissionais liberais (pulverização) é essencial a avaliação do impacto produtivo nesta dimensão. $\mathrm{O}$ armazenamento das informações acerca da qualidade dos serviços prestados por estes (sub)contratados permitirá o pleno exercício das garantias, assim como também, uma possibilidade de futura nova contratação destes para realização de serviços de manutenção. O BIM deverá ser o banco de informações a ser compartilhado com os donos de obra para efetivação da gestão da manutenção ao longo do ciclo de vida do edifício. A correta manutenção dos sistemas garantirá, entre inúmeras vantagens, por exemplo uma maior eficácia dos sistemas de controlo térmico, evitando um maior gasto energético, e dessa forma contribuindo para a sustentabilidade (6D).

Os dados recolhidos nos ensaios realizados incluem (i) a classificação da atividade efetuada, (ii) o respetivo início e fim (data e hora) e (iii) a identificação do trabalhador. Não foram recolhidos dados de localização, que exigiriam um tipo distinto de sensores (wearables e/ou fixos). Refere-se, porém que esta informação espacial poderá ser associada aos dados recolhidos, quer usando coordenadas geométricas provenientes dos sensores espaciais, quer 
usando a identificação do espaço onde a tarefa se realiza ou do elemento construtivo relevante mais próximo, neste caso recorrendo à informação 3D proveniente do modelo BIM. Embora esta informação possa ser associada a um ficheiro BIM, nomeadamente em formato IFC, usando a classe IfcTask e a relação IfcRelAssignsToProcess, considera-se que esta não é necessariamente a melhor opção em cenário real. Com efeito, o volume de dados recolhidos pelos sensores resultaria num modelo de grande dimensão, dificultando operações de edição e de consulta. Assim, sugere-se em alternativa armazenar a informação referida numa estrutura de dados independente (por exemplo, em formato JSON, XML ou SQL), que poderá ser relacionada com o modelo e com os dados de planeamento. A manutenção desta estrutura de dados, independente mas relacionável com a do modelo 3D, é compatível com a noção de information container prevista na norma ISO 19650.

\section{Considerações Finais e Trabalhos Futuros}

O uso das informações pertinentes ao trabalho desenvolvido pelos trabalhadores (WD - Workers Dimension) permite uma análise pormenorizada dos processos produtivos. De forma que potencia a melhoria das condições de trabalho. Aumentos na qualidade da construção podem levar a uma maior durabilidade sem haver necessidade de nova intervenção no edifício. Além disso, a diminuição do custo e tempo na construção do edifício devidos ao aumento da produtividade, podem levar a um excedente monetário a ser parcialmente aplicado em novas tecnologias de manutenção do edifício, como por exemplo: tecnologias de monitorização encapsuladas nos elementos constituintes do edifício e através de modelos BIM LOD 500.

Essa informação tem que ser previamente recolhida e tratada. Com base no estudo de caso apresentado, potencialmente no melhor cenário pode-se atingir uma acuracidade entre $86 \%$ a 94\% na deteção da tarefa a ser executada, o que indica um ótimo desempenho dos algoritmos de tratamento automático da informação a ser incorporada nos usos BIM. Contudo, é preciso salientar que, a aplicação bem sucedida de machine learning requer um número considerável de observações de diferentes tipos de cenário, o que poderá ser um obstáculo para prever a ocorrência de determinadas situações invulgares.

Trabalhos futuros serão realizados em situações reais de obra, na busca de avaliar o desempenho dos dispositivos e também o nível de aceitabilidade do uso destes por parte dos trabalhadores da mão de obra direta. Também, com uma maior variedade e quantidade de dados será possível aferir o potencial prático do processamento em machine learning quanto aos aspetos não apenas inerentes à produtividade mas também quanto à segurança e qualidade.

\section{Referências}

[1] McKinsey, 'Reinventing Construction: a Route To Higher Productivity', 2017.

[2] M. Farmer, 'The Farmer Review of the UK Construction Labour Model: Modernise or Die', 2016.

[3] McKinsey, 'The new age of engineering and construction technology', 2017.

[4] McKinsey, 'Imagining construction's digital future', 2016.

[5] A. Aryal, A. Ghahramani, and B. Becerik-Gerber, 'Monitoring fatigue in construction workers using physiological measurements', Autom. Constr., vol. 82, pp. 154-165, 2017. 
[6] X. Li, P. Wu, G. Q. Shen, X. Wang, and Y. Teng, 'Mapping the knowledge domains of Building Information Modeling (BIM): A bibliometric approach', Autom. Constr., vol. 84, no. October 2016, pp. 195-206, 2017.

[7] L. Ding, Y. Zhou, and B. Akinci, 'Building Information Modeling (BIM) application framework: The process of expanding from 3D to computable nD', Autom. Constr., vol. 46, pp. 82-93, 2014.

[8] R. Charef, H. Alaka, and S. Emmitt, 'Beyond the third dimension of BIM: A systematic review of literature and assessment of professional views', J. Build. Eng., vol. 19, no. May, pp. 242-257, 2018.

[9] D. Calvetti and M. C. Gonçalves, 'Estado de arte da avaliação da produtividade na construção através de ferramentas e dispositivos', Construção 2018. Porto, Portugal, 2018.

[10] P. Kumari, L. Mathew, and P. Syal, 'Increasing trend of wearables and multimodal interface for human activity monitoring: A review', Biosens Bioelectron, vol. 90, pp. 298 307, 2017.

[11]J. Ryu, J. Seo, H. Jebelli, and S. Lee, 'Automated Action Recognition Using an Accelerometer-Embedded Wristband-Type Activity Tracker', J. Constr. Eng. Manag., vol. 145, no. 1, pp. 1-14, 2019.

[12]L. Bao and S. S. Intille, 'Activity recognition from user-annotated acceleration data', in Second International Conference, PERVASIVE 2004, 2004, pp. 1-17.

[13] N. D. Nath, T. Chaspari, and A. H. Behzadan, 'Automated ergonomic risk monitoring using body-mounted sensors and machine learning', Adv. Eng. Informatics, vol. 38, no. August, pp. 514-526, 2018.

[14] W. Tao, Z. H. Lai, M. C. Leu, and Z. Yin, 'Worker Activity Recognition in Smart Manufacturing Using IMU and sEMG Signals with Convolutional Neural Networks', Procedia Manuf., vol. 26, pp. 1159-1166, 2018.

[15] H. F. Nweke, Y. W. Teh, M. A. Al-garadi, and U. R. Alo, 'Deep learning algorithms for human activity recognition using mobile and wearable sensor networks: State of the art and research challenges', Expert Syst. Appl., vol. 105, pp. 233-261, 2018.

[16] T. Sztyler and H. Stuckenschmidt, 'On-body localization of wearable devices: An investigation of position-aware activity recognition', in 2016 IEEE International Conference on Pervasive Computing and Communications, PerCom 2016, 2016, pp. 1-9.

[17] S. H. Cha, J. Seo, S. H. Baek, and C. Koo, 'Towards a well-planned, activity-based work environment: Automated recognition of office activities using accelerometers', Build. Environ., vol. 144, no. April, pp. 86-93, 2018.

[18] PMI, Um guia do conhecimento em gerenciamento de projetos (Guia PMBOK). Quinta edição. Newtown Square, Pa.: Project Management Institute, Inc., 2013.

[19] T. O. Olawumi, D. W. M. Chan, J. K. W. Wong, and A. P. C. Chan, 'Barriers to the integration of BIM and sustainability practices in construction projects: A Delphi survey of international experts', J. Build. Eng., vol. 20, no. July, pp. 60-71, 2018.

[20] PMI, Um guia do conhecimento em gerenciamento de projetos (Guia PMBOK). Sexta edição. Newtown Square, Pa.: Project Management Institute, Inc., 2017. 